\title{
Back to basics
}

\author{
Successful translational research requires a solid understanding of the systems involved and their normal functions, \\ which only basic research is equipped to provide.
}

the t has been 13 years since the end of the "Decade of the Brain," and the neuroscience community is contemplating another decadelong federal project: the BRAIN initiative. Many neuroscientists are looking to the technical innovations that this initiative will stimulate and the exciting developments that will ensue. After all, a lot can happen in 10 years. Neuroscience has seen some incredible leaps in the last decadewe've come to appreciate the incredible plasticity of the adult brain and gained a deeper understanding of the role of glia. We've seen the genomes of humans, mice and many other organisms sequenced and come to appreciate the role of epigenetics in behavior and disease. Although there have been exciting breakthroughs in understanding the basic neurobiology behind many phenomena, over this time, there has been an increasing focus on translational research, driven in part by funding priorities. President Obama's budget proposal for 2013 continues to increase funding for applied research, including an additional $\$ 80$ million specifically earmarked for Alzheimer's disease research, often at the cost of funding for basic science. The increasing influence of foundations focused on curing disease is also driving more translational work. The success of translational research, however, is unproven. Although there are certainly success stories, many efforts have failed, at least when judged on the basis of the lack of treatments or cures for diseases that have been produced. A foundation of knowledge about the systems or circuits involved in the disease is essential for translational success. In many areas of neuroscience, we don't yet have such a foundation. Scientists must make the case to the public that basic research is essential to support such clinically minded endeavors; without it, many translational efforts are doomed to fail.

In this age of austerity, it can be difficult to convince members of Congress to support basic research. This was made apparent recently by criticisms of the National Science Foundation's grant review process, and the drafting of a bill by Lamar Smith (a Republican Congressman from Texas) to require that National Science Foundation-funded grants be focused on problems "of utmost importance to society." In the short term, translational studies are an easier sell to politicians, but scientists should be wary of couching their arguments in favor of funding research in such terms. If you promise cures, the public sees anything less as a failure, even if valuable knowledge comes out of the work despite the lack of a cure. Although it's more difficult in the short term, arguing the merits of basic research will benefit scientists in the long term. After all, many medically relevant discoveries have come seemingly out of left field: observations of naked mole rat colonies, for example, recently led to the identification of signaling pathways that mediate their resistance to cancer, a discovery that could have profound implications for human health. Studies such as this are only made possible when value is placed not only on work specifically designed to look for cures to diseases but also on work that aims to understand the fundamentals of biology in all of its diverse forms.
The study of psychiatric disease has provided a prime example of the importance of understanding basic mechanisms and circuitry before launching into translational studies. Despite concerted research efforts aimed at identifying new antidepressant drugs, no mechanistically new drugs have been successfully developed in the last several decades. This lack of progress can be attributed, at least in part, to the lack of representative animal models of the disease. Because we don't truly understand the pathophysiology of depression, we can't generate valid animal models, and without models in which to test them, new drugs can't be developed. Recent changes in policy at the National Institute of Mental Health (NIMH) may begin to address this problem with the so-called Research Domain Criteria. Rather than relying on imperfect symptom-based diagnostic categories, the NIMH is now encouraging researchers to study "basic dimensions of functioning." These basic dimensions, for example, fear circuitry or working memory, are defined by knowledge of the underlying biology of fundamental elements of the nervous system and may cut across diagnostic boundaries. For example, genetic studies have shown that some gene variants increase risk of developing multiple psychiatric diseases, such as schizophrenia and autism, which were not previously thought to be related. Studies designed under these guidelines could provide the basic understanding of emotional and cognitive processes necessary to determine what goes wrong with these systems in psychiatric disease. This new approach, if it succeeds, could serve as a model for other fields, including neurodegenerative disease research, where some of the same symptom heterogeneity and overlap between diseases is also present.

As we look ahead, it's clear that the President's BRAIN Initiative will influence the course of neuroscience in the next decade. As with the Human Genome Project before it, it seems to be the ultimate basic science endeavor: an effort to understand the basic structure and function of the brain. Yet at least some involved in the project have touted it as a roadmap to curing psychiatric or neurological disease. Although the type of research likely to be funded by this initiative would undoubtedly be valuable to support future translational work, it's dangerous for neuroscientists to over-promise the medical advances that will result from it. If such cures fail to emerge, the public will judge the project harshly, no matter the benefit to our understanding of how the brain works. Instead, scientists need to make the case to the public and politicians that basic research like the BRAIN initiative is valuable and is a necessary precursor to successful translational work. Whatever emerges from the BRAIN initiative, whether advances in our understanding of the basic functioning of the brain or cures for disease, the next few years are sure to be a captivating time in neuroscience. We look forward to the exciting advances in both basic and translational neuroscience that we're confident will emerge. 